\title{
Bernd Spangenberg, Colin F. Poole, Christel Weins: Quantitative Thin-Layer Chromatography: A Practical Survey
}

\author{
Ian D. Wilson
}

Published online: 27 February 2011

(C) Springer-Verlag 2011

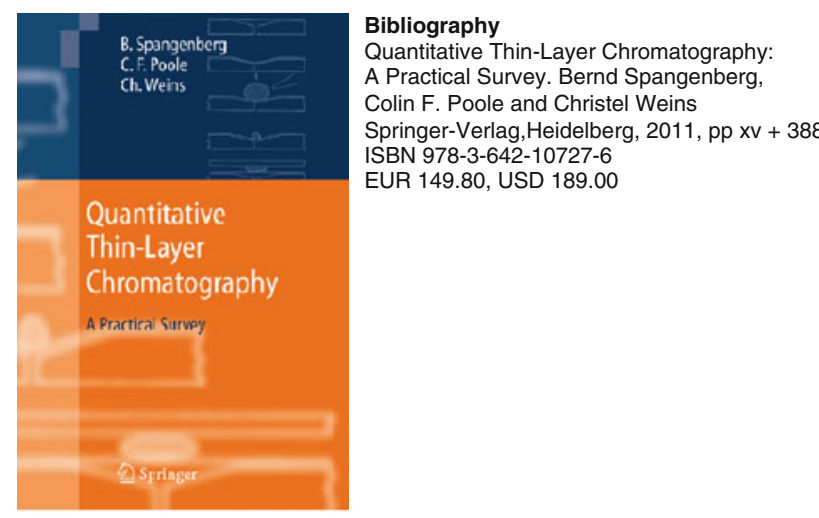

This new book on quantitative thin-layer chromatography, sometimes also referred to as planar chromatography, is a welcome addition to the literature on this useful but rather unglamorous division of separation science. Indeed, whilst still widely used, TLC is generally employed as a qualitative or semi-quantitative technique for rapid analysis of samples where full quantification is perhaps less important than identification. The publication of an up-to-date book that reminds chromatographers and analytical chemists of the quantitative possibilities of the technique is therefore to be welcomed. The book consists of some 14 chapters that cover everything from the history of the technique, through the theoretical aspects that must be studied for TLC to be properly understood before considering the practical aspects such as stationary and mobile phases, sample preparation, plate development, detection and validation. Each chapter is self contained and appropriately referenced.

I. D. Wilson ( $\square)$

Maccesfield, Cheshire, UK

e-mail: Ian.Wilson@astrazeneca.com
Those unfamiliar with instrumental methods of TLC will, I think, be surprised by the relative sophistication of some of the devices available to the modern planar chromatographer. As this volume illustrates, precise and careful sample application can now be performed via computer controlled automated instruments that can spray the sample onto a range of stationary phases almost as wide as those available for column liquid chromatography. Following sample application, carefully controlled plate development using methods such as traditional capillary flow, or various modes of forced flow development, the spots or bands of analyte can subsequently be quantified with a variety of detectors ranging from "traditional" UV/visible reflectance instruments, through diode array detectors, and imaging via video-densitometric detectors. Fluorescence detection also provides a convenient means of quantification for compounds and UV, visible and fluorescence modes can also be enhanced by the whole range of post chromatographic derivatization reactions possible on TLC plates, and often denied to the column chromatographer. In addition to these conventional detection systems, near infrared, infrared, surface-enhanced Raman and radioactivity detection as well as direct and indirect MS and on-plate "bioassays" can be performed.

Overall, this is a readable, well-produced and nicely illustrated volume (with numerous colour pictures) and a moderately useful index of approximately 14 pages. It can be highly recommended to anyone wishing to reacquaint themselves with a once-familiar technique that they have lost touch with. Similarly, for a newcomer to TLC wanting to get up to date with the potential offered by twenty-first century instrumental TLC, this is book provides a good place to start. 